\title{
3D Characterization of Sand Particle-to-Particle Contact and Morphology
}

\section{Andrew M. Druckrey ${ }^{1}$, Khalid A. Alshibli ${ }^{2}$, and Riyadh I. Al-Raoush ${ }^{3}$}

\begin{abstract}
Particle morphology, orientation, and contact configuration play a significant role in the engineering properties of granular materials. Accurate three-dimensional (3D) characterization of these parameters for experiments has historically proven difficult, especially in the context of particle contact with small particle size. This paper describes a computer code that was developed to analyze 3D images of granular materials to measure particle lengths (size), volume, surface area, global centroid location and orientation; it also provides a method to calculate particle contact location and orientation. Measurements from the proposed code can define a state of the granular material's fabric that can be used as input for micro-mechanics based constitutive models and to validate numerical discrete element simulations. A fabric tensor and its evolution is calculated based on experimental contact normal vectors that were extracted from SMT imaging of an axisymmetric triaxial compression experiment on a natural silica sand known as F-35 sand.
\end{abstract}

\section{Key Words: Normal to contact, Fabric, synchrotron computed tomography, granular materials}

\footnotetext{
${ }^{1}$ Graduate student, Dept. of Civil \& Env. Engineering, 325 John Tickle Building, University of Tennessee, Knoxville, TN

${ }^{2}$ Professor, Dept. of Civil \& Env. Engineering, 325 John Tickle Building, University of Tennessee, Knoxville, TN 37996, USA, Tel. 011-865-974-7728, Email: Alshibli@utk.edu

${ }^{3}$ Associate Professor, Dept. of Civil \& Arch. Engineering, Qatar University, Qatar, Email: Riyadh@qu.edu.qa 


\subsection{Introduction}

Granular materials are composed of discrete particles that interact with each other in a complex fashion. Particle gradation, mineralogy, morphology, and contacts are the major factors that contribute to the fabric of granular materials and to rather a complex interaction between particles and mechanical properties, which is difficult to analyze at the micro-scale. Accurate characterization of particles and their interaction is essential for the development of micromechanics based constitutive models for granular materials. X-ray computed tomography (CT) image acquisition and processing has greatly advanced the quantification of different engineering properties of granular materials.

Extensive research has been published to quantify individual particle characteristics such as particle size, shape, spatial coordinates, and orientation using both 2D and 3D CT imaging (e.g. Thompson et al. (2006); Al-Raoush (2007); Cox and Budhu (2008); Bloom et al. (2009); Cnudde and Boone (2013); Alshibli et al. (2014)). Furthermore, research focusing on fabric evolution while subjecting granular mass to in situ loading has been investigated using x-ray CT. For example, Hasan and Alshibli (2010) used synchrotron micro-computed tomography (SMT) to image a plane strain specimen, quantified particle orientation within the shear band, assessed particle-to-particle interaction. Hall et al. (2010) acquired multiple in situ CT images of a triaxial specimen composed of Hostun RF sand, discretized particles, and analyzed discrete and continuum deformation within the specimen. Andò et al. (2012) expanded on Hall's approach and developed ID-Track computer code to quantify particle kinematics at multiple loading stages for many particles within triaxial specimens composed of Hostun RF (angular) and Caicos Ooid (rounded) sands. Similarly, Druckrey and Alshibli (2014) developed an algorithm to track kinematics of ASTM 20-30 sand based on SMT images during triaxial testing. 
Recently, particle-to-particle contact and evolution of contact are considered as important factors in fabric evolution and force transmission in granular materials. Few researchers have developed methods to determine particle coordination number $(\mathrm{CN})$ and track the $\mathrm{CN}$ of individual particles as test progresses based on CT and SMT imaging. However, identifying particle contact and its spatial coordinates do not provide full insight into how particles interact with neighboring particles. A more comprehensive definition would include location as well as orientation of contact in 3D space. Moreover, calculating the location of contact and the normal and tangential directions to contact would provide the necessary measurements to quantitatively define the contact between two particles. Few experimental studies have been proposed to quantify particle contact orientation. Viggiani et al. (2013) presented a method to determine the orientation of contact based on CT particles composed of voxels ( $\sim 5000$ voxels per particle) by fitting a plane to the contact voxels between two particles, in which the distribution of the normal unit vector varies based on the type of algorithm that was used to separate particles. Vlahinić et al. (2014) used mathematical level set functions on the greyscale 3D x-ray CT image to determine the orientation of contact.

Directional data, such as contact normal vectors, can be characterized by fabric tensors and several definitions have been proposed in the literature. Oda (1972) discussed the initial fabric of granular materials and how it relates to their mechanical properties. Kanatani (1984) established methods for mathematically quantifying tensors that describe directional data distributions and applied them to characterize inter-particle contact distribution in granular materials. Many researchers have adopted a form of Kanatani's fabric tensor of the first kind, or moment tensor, and have applied it to characterize the fabric of granular materials based on various geometrical features. Oda et al. (1985) used fabric tensors to investigate stress-induced anisotropy in granular 
masses of biaxial compression tests of oval rods using photo-elastic pictures and contact normals. Others have used discrete element method to investigate effects of granular material fabric on various properties (e.g. Yimsiri and Soga (2010), Theocharis et al. (2014), Yang et al. (2014)). Fonseca et al. (2013) used CT and image analysis tools to investigate stress transmission in granular materials by extracting cores from triaxial specimens impregnated with resin.

The accuracy of the current methods relies heavily on the resolution of the CT/SMT images, especially sharpness at particle boundaries (phase contrast). Blurry particle edges/contacts make it difficult to determine voxels that belong to a particular particle during particle separation. Industrial x-ray CT imaging produces unclear particle boundaries and questionable contact orientations. This paper presents a method that utilizes high-resolution 3D SMT image analysis to quantify individual particle morphology and defines contact that includes contact location and orientation. It includes an experimental assessment of fabric evolution of F35 sand undergoing axisymmetric triaxial compression during in situ SMT imaging using fabric tensor techniques.

\subsection{Image Acquisition}

In the last three decades, $\mathrm{x}$-ray $\mathrm{CT}$ has emerged as a powerful non-destructive 3D scanning technique to visualize the internal structure of materials. It involves probing a specimen mounted on a rotating stage from different angles and collecting the attenuated x-ray using a detector system. Post-scan computer reconstruction and processing involve creating multiple slices through the scanned specimen that can be stacked to generate a $3 \mathrm{D}$ volume rendering of the specimen. In this paper, SMT images of silica sands were acquired at the Bending Magnet Beamline 13D (13 BMD) at the Advanced Photon Source (APS), Argonne National Laboratory 
(ANL), Chicago, USA. SMT imaging offers many advantages when compared to industrial x-ray CT imaging, which is well documented in the literature (e.g. Baruchel et al. (2006), Brunke et al. (2008), Cnudde and Boone (2013)). The main advantages include an almost parallel beam and high brilliance (which results in high photon flux on the specimen) yielding a much better image resolution and signal-to-noise ratio in the images (Baruchel et al., 2006). High flux monochromaters can tune the beam to monochromatic radiation for the desired energy level to suit specimen size and attenuation level.

Specimen sizes at beamline $13 \mathrm{BMD}$ can range from a single particle with a resolution on the order of $1-2 \mu \mathrm{m} /$ voxel to a $10 \mathrm{~mm} \times 20 \mathrm{~mm}$ miniature triaxial test with resolutions of $8-11$ $\mu \mathrm{m} /$ voxel. Specimens can range from dry to fully saturated and can include a variety of particle morphologies. Specimens were rotated at a constant velocity and radiograph images are obtained by the detector at $0.2^{\circ}$ rotational increments. More information on the setup of beamline 13BMD can be found in Rivers et al. (2010).

\subsection{SMT Image Analysis}

\subsection{Particle Identification}

3D SMT images must be post-processed to transform the grayscale SMT image to a binary image and eventually to an image where each particle is individually identified and labeled. The labeled image will be used to compute particle properties as well as to characterize particle contacts. Accurate identification and calculations of individual particle morphology parameters and particle-to-particle association of the bulk material can then be defined and analyzed to provide critical parameters to feed in micro-mechanics-based constitutive models as well as provide validation for numerical modeling. 
Grayscale SMT images were imported into Avizo Fire software using the built-in raw data read option (Fig. 1a). To remove noise within the image while preserving particle edges and enhancing the contrast of the edges, an anisotropic diffusion filter was applied to the grayscale image. The anisotropic diffusion algorithm compares the value of each voxel with its six facecentered neighbors and diffuses the voxel as long as the difference does not exceed the input diffusion stop threshold value, which was determined by a parametric study and error analysis (Fig. 1b). The resulting image is a smoothed grayscale image with sharp particle edges between the solid particle material and the surrounding medium.

The next step in image processing was to segment the grayscale image in order to separate particles from the surrounding medium. Segmentation by interactive thresholding was employed to create a binary image assigning a voxel value of 1 for sand (having a higher x-ray attenuation) and 0 for the surrounding medium (usually air, which has a lower attenuation). The interactive thresholding produced a binary image that represents particles well. The binary image clearly shows particle contacts to the scale of image resolution. In other words, if particles are physically in contact at a point as small as the resolution, the binary image will represent this contact point. The segmentation process leaves small holes within some particles or small fragments of noise within the air surrounding particles. Further image processing using morphology operations on the image was implemented to remove noise from the images. First, Avizo Fire's 3D algorithm, Fill Holes, which fills all holes that are completely surrounded by material (solids) without affecting particle surfaces. Next all small noise must be removed so that it is not mistaken for actual particles; this is usually only a few voxels in volume within the surrounding air. The opening command was used to clean up other noise without affecting overall particle shape. The 
resulting binary image is depicted in Fig. 1c and will be used later in image processing as a mask to ensure the final labeled image is accurate.

Now that the binary image accurately represents particles and surrounding void space, contacts between particles must be removed in order to label each particle. Avizo Fire's Separate Objects module is a combination of watershed, distance transform, and numerical reconstruction algorithms that can accurately remove the small area of contact between particles. It works well when particles are mono-sized and generally convex and results in separating the binary particles from each other similar to the image in Fig. 1d. Next, the Border Kill module was applied to the image to remove all of the voxels connected to the edge of the 3D image. Finally, the particles were individually labeled from 1 through the number of particles, then particle-to-particle contact was reestablished. To omit the unnecessary expanded portions of the particle (regions of the particle boundary that were expanded excluding contact points), the image was masked with the binary image (Fig. 1c) that precisely represents particle morphology and fabric. The resulting image (Fig. 1e) accurately represents individually labeled in-contact particles.

Each particle label consists of connected voxels that have the same value, and each voxel occupies a cubical "point" in 3D space within the image. As a result of the high resolution and clarity of SMT images, the 3D voxel representation of particles captures morphology very well. Although measurements as small as surface texture cannot be accurately quantified using current SMT imaging, other properties such as volume, size, surface area, and contact can be accurately defined as demonstrated in the following sections. These properties were calculated using a computer code that was developed by the authors. The code is divided into two "loops" one to define individual particle properties followed by a double loop to define contact properties. First, the labeled image was read into memory as a 3D matrix, and each particle label voxel was 
searched for with the first loop. In each iteration of the loop, the physical location of each particle's voxels were stored in an $n \times 3$ matrix, where $n$ is the number of voxels belonging to a particular particle on that iteration of the loop. The three columns of the particle matrix are the $\mathrm{x}$, $\mathrm{y}$, and $\mathrm{z}$ coordinates of voxels that constitute the particle and can be easily used to calculate particle physical properties. For example, the volume of each particle was calculated as the summation of all voxels within the particle, or simply the length of the $n \times 3$ matrix. Particle volume was added to a vector containing all particle volumes from the preceding iterations after each iteration of the loop. This first loop also stores information about each particle surface that will be passed on to the contact definition loop.

\subsection{Particle Center of Mass}

Center of mass of each particle is the 3D volumetric center, or center of gravity in $\mathrm{x}, \mathrm{y}$, and $\mathrm{z}$ directions. The 3D matrix of particle voxel locations was used to determine the center of mass. An average spatial location was calculated by summing voxel locations in each direction (each column of the $\mathrm{n} \times 3$ voxel location matrix) divided by the total volume of the particle (after Al-Raoush (2007)):

$$
\begin{aligned}
c g_{i} & =\frac{\sum_{i} i f(i, j, k)}{\text { volume of particle }} \\
c g_{j} & =\frac{\sum_{j} j f(i, j, k)}{\text { volume of particle }} \\
c g_{k} & =\frac{\sum_{k} k f(i, j, k)}{\text { volume of particle }}
\end{aligned}
$$

where $c g_{i}, c g_{j}$, and $c g_{k}$ are the global center of mass of each particle $f(i, j, k)$ in the $\mathrm{x}, \mathrm{y}$, and $\mathrm{z}$ directions, respectively. An accurate determination of center of mass is essential in investigating particle kinematic behavior during shearing of granular materials. The proposed method 
determines the exact location of the particle center of mass in 3D space regardless of particle translation and/or rotation during testing and can provide valuable insight into behavior of granular material under any type of loading condition.

\subsection{Particle Surface Area}

Particle surface area influences the behavior of granular materials in many ways. Surface area can be used to quantify morphology (sphericity and roundness) of particles, which affects the strength and deformation characteristics of granular materials. Traditionally, particle surface area has been difficult to measure physically for small particles. Emergence of SMT imaging and analysis has greatly enhanced the ability to perform surface area measurements on small particles. The high resolution of SMT images provides virtual particles that very accurately portray particle shape via voxels, and measurements of surface area are not as elusive as before. To calculate the surface area of a particle, one must determine which voxels are on the boundary (surface) of the particle. Each voxel of the particle was analyzed to determine if it has any free faces in each iteration of the loop (free faced voxels have faces not directly adjacent to another voxel that belongs to the same particle but rather either next to void space or another particle). To accomplish this analysis, each voxel of a particle was individually analyzed to determine if any part of that voxel was on the particle's surface. There are three situations where this can occur. The voxel face, as discussed earlier, can be directly on the surface, and there are six possible situations (six cubic faces) that occur where the face could be on the surface of the particle (Fig. 2a). A voxel edge can be on the surface, which has 12 possible occurrences for the 12 edges of a cubic voxel (Fig. 2b), or one of the eight voxel corners can be touching the surface (Fig. 2c). 
There are 26 total situations in which a voxel within a particle can be touching the surface. Representing these situations numerically entailed creating 26 matrices of length $n$, recalling that $\mathrm{n}$ is the total number of voxels within the particle. Six of the matrices store locations of the six voxels surrounding each voxel face. Twelve of the matrices store locations of the voxels connected to edges of each voxel. Eight of the matrices store locations of the voxels connected to corners of each voxel. Among all 26 matrices, the location of all voxels surrounding each particle voxel are known and can be searched to determine what, if anything, occupies them. If they do not contain the label ID of the particle being analyzed (i.e., they contain air or a different particle's voxel), then the voxel they surround is determined to be connected to the surface of the particle. Surface voxels are stored, and Fig. 3 displays an example of voxels located on a particle surface. Surface area is calculated as the summation of voxels with free faces on the surface of the particle (excluding those with only corners and edges on the surface). Another matrix that includes the location of all voxels surrounding the particle, or a shell around the particle, is also stored for further use in contact determination. These are the voxels that come into contact with the particle at all voxel faces, corners, and edges.

A sphere made up of voxels (diameter of 151 voxels) was created and analyzed with the code to check its accuracy in calculating particle surface area. The code result was 71880 voxels $^{2}$, compared to a theoretical value of 71631.45 voxels $^{2}$, resulting in a $0.3 \%$ error between the calculated and theoretical values. The slight deviation is attributed to the voxel nature of the sphere. 


\subsection{Particle Lengths and Orientation}

Orientations and sizes of particles in granular materials play a critical role in determining their behavior. Mostly, particle orientation and shape determines the initial fabric and its evolution during shearing. It contributes to the strength and dilatancy of granular materials as well as other engineering properties. Three principal lengths of a particle in $3 \mathrm{D}$ are defined as orthogonal and along the short, intermediate, and long axes of the particle.

Lengths and orientation of each particle are determined using the concept of Principal Component Analysis (PCA) on the multi-variant set of points that make up the particle voxels. PCA uses linear correlation between the points and applies a trend line to the long axis of the data by minimizing distances $(\mathrm{x}, \mathrm{y}$, and $\mathrm{z})$ between the data points and the line using singular value decomposition. The principal component coefficients describe the global angle of the trend line determined by PCA, and the principal component scores represent the fitted location of the voxels on the 1D trend line in principal space. The lengths of a particle were calculated as the difference between the maximum and minimum scores along each of the trend lines. There are two principal component coefficients in a $2 \mathrm{D}$ image of a particle governed by the $\mathrm{x}$ and $\mathrm{y}$ location of the points. For example, Fig. 4 displays a 2D section of a particle composed of 6810 voxels, along with the results of 2D PCA displayed via a projected view that passes through the particle centroid. Principal component coefficients represent the global orientation of the particle lengths (vector directions shown in Fig. 4) and lengths of the long and short axes are based on differences in extreme score values (extreme points fitted on trend line). The PCA method accurately represents how particles would be measured physically (twice the length of vectors shown in Fig. 4) because it is the difference between extreme points of a particle as if measured 
by a micrometer. It is important to note that particle lengths obtained from PCA do not necessarily pass through the particle center of mass for non-spherical particles.

Three orthogonal principal components are defined along the three principal axes of the particle in 3D case, representing the long, intermediate, and short axes of a particle. Orientations of the three principal axes were calculated as the principal component coefficients of the PCA dataset. The long axis length of the particle is the difference between the extreme points that make up the first principal component. The intermediate and short axes lengths were calculated in the same manner on the second and third principal components, respectively. PCA yielded accurate measurements of the orientations and principal lengths of each of the particles in 3D. Figure 5 displays a 3D surface of a particle along with vectors that identify the particle's global orientation and length. The red, blue, and magenta vectors represent the orientations of the long, intermediate, and short axes of the particle, respectively (displayed through the particle center of mass). Principal components are orthogonal to each other and globally oriented based on the statistical computation by PCA. Global orientations of particles were extracted and can be used to represent fabric of granular materials as well as to calculate particle rotation during shearing.

A 3D SMT image of a triaxial experiment on ASTM 20-30 Ottawa sand was analyzed using the proposed code as a demonstration dataset. Resolution of the SMT image was 10.85 micron/voxel, and the image consisted of 3400 particles. ASTM 20-30 Ottawa sand has a particle size between US sieves \#20 $(850 \mu \mathrm{m})$ and \#30 $(600 \mu \mathrm{m})$; therefore, particle sizes based on the SMT image should fall within that size range. Particle size distribution based on SMT image analysis is depicted in Fig. 6, which shows a normal statistical distribution having sizes mostly between 600 and $800 \mu \mathrm{m}$ with a small percentage larger than $850 \mu \mathrm{m}$. Particle size is determined from the SMT images based on the average of the small and intermediate lengths, 
which is used to compare particle size to mechanical sieving. Averaging these lengths better represents the physical process of mechanical sieving; for example, a particle's small axis length might fit through sieve mesh where its orthogonal intermediate axis might not. Based on this, a small portion of the particles are larger than what is expected.

\subsection{Coordination Number and Particle-to-Particle Contact Definition}

Identification of contact between particles involves analysis of the boundary shells that were described earlier for each particle. A matrix was generated that initially contained all voxels surrounding the particle surface (the shell around the particle determined from the first loop). The voxels of the shell that do not contain another particle (the void space) are then removed from the matrix based on the labeled image. What remains in the matrix is the portion of the shell that is occupied by other particles in contact with the particle being analyzed. Furthermore, data can be extracted on each particle that is in contact with the particle being analyzed by searching the original labeled image for the particle ID whose voxels occupy that space on the boundary. Coordination number is calculated as the total number of unique particle IDs within that matrix, which is the total number of particles in contact with the particle being analyzed.

The contact between particles was computed within the second loop of the code. Definition of contact between two particles consists of identifying which voxel faces, corners, and/or edges share a mutual face, corner, and/or edge with a neighboring particle and calculating the location and directions of that contact. The outer loop cycles through each particle and collects the spatial location indices of contact in a vector as well as a vector contains particle numbers which are in contact with the particle being analyzed. These two vectors were passed to inner loop that calculated contact properties. The code searches the labeled image to match a particle number to 
its contact location, and the number of voxels that defines the contact between two particles is a representation of a surface between them. Even with high-resolution SMT images, this contact surface is only relative to the image and cannot be quantified with any real physical meaning. Figure $7 \mathrm{~b}$ displays an example contact (shown in red) between two voxel particles. Contact location was calculated by adding the voxel locations in each direction $(\mathrm{x}, \mathrm{y}, \mathrm{z})$ and dividing them by the total "area" of contact, similar to the calculation of particle centroids.

Contact between two particles is not always a single continuous surface. Roughness of particle surface or complex morphology could lead to several contact points, especially in very angular or rough granular materials. Considering that particle contacts may not be continuous, contact voxels do not lie in a plane as evident from Fig. 7c. Although this definition of contact does not take into account the discontinuity of the contact surface, some researchers considered a global average of the voxel locations in calculating the contact and orientation. Contact area is relatively small and the global definition of contact between two particles will produce an overall average of the contact. This provides a good basis for determining fabric of a granular material, but may not prove as valuable for particle fracture assessment, since particle fracture depends heavily on contact and inter-particle forces. To define the contact between two particles, the 3D global direction of contact must be known as well as its location. The normal and tangent vectors to contact can play a significant role in determining how the particles will interact with each other upon loading and shearing. For example, if the loading direction is parallel to the vector normal to contact, then the forces transmitted between particles will be higher than if the loading direction was perpendicular to the normal contact vector. A statistical analysis was conducted on the contact voxels using PCA to determine contact orientation. The first and third principal coefficients represent the tangent vector to contact (first principal component along long axis or 
plane of contact voxels) and the normal vector to contact (third principal component along shortest axis of voxels). Essentially, the 3D normal and tangent vectors are on the best fit plane as determined by PCA. Fig. 8 shows example normal and tangent contact vectors located at the center of contact for the particles depicted in Fig. 7. PCA of the contact voxels provides the global orientation of contact and insight into how particles interact with each other when they are loaded. Defining the global contact location and normal between particles is useful in determining force transfer through the granular material. Even if the contact is discontinuous, PCA determines the statistical center and normal/tangent vectors of contact. It represents the location and direction in which force will statistically be transmitted between particles, which is valuable input in micro-mechanics based constitutive models.

The code flow chart is shown in Fig. 9. The code consists of two main loops. Input for the first loop is the labeled image, and volume, center of mass, surface area, coordination number, lengths and orientations are calculated for each particle and stored as output. Coordination number and contact voxel location are used as input for the second loop, which is a double for loop. The outer loop collects the contact locations for a particle, as well as particle numbers that are in contact with the particle being analyzed. The inner loop determines the contact orientation with PCA and the centroid of contact. Contact centroid and orientation is stored for each contact of each particle.

Some difficulties were encountered in calculating contact between particles within the code. PCA requires more than one pixel being in contact for calculation of the three principal components. When contacts are limited to only one voxel, whether from pieces of noise within the image or a physically small contact (less than image resolution), those points are omitted from the analysis. They make up a negligible percentage of the total contacts within any SMT 
image using the current image processing analysis. Another situation that can possibly occur and cause errors in defining contact orientation is when a particle has an extremely concave surface and wraps around another particle. This type of contact does not accurately fit a plane to the contact voxels well; however, PCA statistically determines the best fit contact orientation. The types of sand used in analysis with this code can be chosen so that this error is not likely to occur.

\subsection{Contact orientation of F-35 sand during axisymmetric triaxial compression}

Many definitions of fabric for granular material are reported in the literature using various geometrical aspects of the material such as contact normal vectors, branch vectors (vector connecting centroids of contacting particles), particle orientation and void space. This paper investigates the evolution of contact normal during axisymmetric triaxial compression experiment on F-35 sand from in situ SMT images. The experiment was conducted on $10 \mathrm{~mm}$ diameter x $20 \mathrm{~mm}$ height cylindrical dense specimen (relative density $=90 \%$ ) that was subjected to $400 \mathrm{kPa}$ confining pressure. Further description of the sand, experimental setup, and experiment can be found in Alshibli et al. (2014), Druckrey and Alshibli (2014) and Tordesillas et al. (2015). SMT images were acquired at axial strains of $0,1,2,3.5,5,7,9$ and $17.5 \%$ and processed by the previously described methods. The SMT images had a resolution of 11.18 $\mu \mathrm{m} / \mathrm{pixel}$ and the principal stress ratio (PSR) versus axial strain for the experiment is depicted in Fig. 10. Peak stress is reached at $\sim 5 \%$ axial strain and critical state was reached at approximately $13 \%$ axial strain.

Normal contact vectors were converted into spherical coordinate system with a rotated coordinate system in order to represent data in the loading direction (z-direction) with a full rose 
diagram ( 0 to 360 degrees, rather than 0 to 180 degrees). YZ direction was chosen in this paper due to limited pages of the paper and the normal contact vector and branch vector distributions are shown as rose diagrams in Fig. 11 for axial strains of $0 \%, 2 \%$ (pre-peak stress state), 5\% (peak stress state), and 17.5\% (critical state). Before loading, contact normal vectors adduce a directional preference toward the horizontal which is expected as particles lay in the most stable position when they are deposited. As loading progresses, the directional preference of the contact normal vectors gravitates toward the loading direction (z-direction), and at peak and critical states contact normal vectors display a preference to resist the vertical load. This trend of contact normals aligning to resist the load agrees with published research on the evolution of contact normals (e.g. Kanatani (1984), Theocharis et al. (2014)). There is only a small difference between the contact vectors at $5 \%$ axial strain (peak principal stress ratio state) and $17.5 \%$ axial strain (critical state). At the peak state, the shear band has nearly fully developed and rotation and kinematics of particles outside the shear band is relatively constant; meaning all particles outside the shear band are either moving as a rigid body (above shear band) or not moving at all (below shear band). As a result, most particle contact vectors remain relatively constant outside the shear band. Branch vector orientations follow a similar trend, although not as dramatic as contact normal vectors. This is attributed to the angularity of the F-35 particles. If all particles were spherical, contact normal and branch vector distributions would be identical.

Kanatani (1984) fabric tensors were adopted in this paper. Fabric tensors of the first kind, which is the moment tensor of the contact normal vectors for each scan, is a symmetric tensor calculated as (second order):

$$
N_{i j}=\frac{1}{N} \sum_{\alpha=1}^{N} n_{i}^{\alpha} n_{j}^{\alpha}
$$


where $n_{i}^{\alpha}$ is the $\alpha$ th contact normal vector and $N$ is the number of contacts. Fabric tensors of the second kind order two is a deviatoric fabric tensor calculated as:

$$
F_{i j}=\frac{15}{2}\left(N_{i j}-\frac{1}{3} \delta_{i j}\right)
$$

where $\delta_{i j}$ is the Kronecker delta. The second order probability density function (PDF) of the contact normal is:

$$
f(n) \sim \frac{1}{4 \pi} F_{i j} n_{i} n_{j}
$$

Moment tensors, deviatoric fabric tensors, and PDF can be calculated to any even order. Second order descriptions of directional data are limited to ellipsoidal shapes for 3D data, while fourth and higher orders can represent more complex distributions. Completely uniform directional data would be represented by a sphere in $3 \mathrm{D}$, or circle in $2 \mathrm{D}$. Second and fourth order deviatoric fabric tensor surface representations are displayed in Fig. 11 for 3D contact normal vectors. Again, the second order tensor representation is limited to ellipsoidal shape while the fourth order tensor describes the data more accurately. Both orders show how the contact normal vectors are initially distributed more towards the horizontal and shift towards the vertical loading direction to resist the applied load.

To describe the evolution of internal structure anisotropy, the normalized second invariant of the second order deviatoric fabric tensor $\left(F_{I I}\right)$ was calculated at each loading stage, similar to Yang et al. (2014) and Theocharis et al. (2014). Fig. 12 shows the evolution of $F_{I I}$ during shearing of the F-35 sand. Fabric anisotropy increases rapidly during pre-peak stress state and reaches a maximum at peak state. The fabric anisotropy neutralizes and is relatively constant during the post-peak regime, implying that once the peak stress is reached minimal changes in overall contact normal direction occur. 


\subsection{Computational Requirements}

The code was originally developed using MATLAB R2012b for relatively small images. For example, a $300 \times 300 \times 300$ voxel image with 120 particles requires approximately 1 GB of memory and $\sim 2$ minutes to process the data on a workstation with 48 GB RAM. As the images increase in size and the number of particles increases, the computational time increases because the images are loaded into memory and they are searched many times within the code. Some of the large SMT images can be in excess of $1920 \times 1920 \times 2600$ voxels and contain over 100,000 particles. Running the MATLAB version of the code requires hundreds of gigabytes of RAM and several weeks of processing time. For this reason, the code was ported to $\mathrm{C}++$ programming language. Once ported, analysis on the large images required approximately 70 GB RAM and 45 hours of compute time. The $\mathrm{C}++$ version of the code can analyze even the largest images available from current SMT technology.

\subsection{Summary and Conclusions}

The paper presented a method of 3D SMT image analysis to quantify particle morphology as well as particle contacts. High resolution SMT images were obtained and processed using Avizo Fire software. SMT imaging and anisotropic diffusion filtering provided grayscale images with very little noise and clear particle boundaries. The SMT images were processed, and individual particles were labeled with their contact points intact. A code was developed in Matlab and ported to $\mathrm{C}++$ language to analyze the largest labeled images available from current SMT technology. Accurate values of particle volume, surface area, center of mass, lengths, and orientations were computed for individual particles. Contact was defined based on contact voxels 
between particles. Those contact voxels were statistically analyzed using PCA to determine the normal and tangent vectors to contact.

Contact normal distributions were analyzed during in situ SMT imaging of triaxial compression of F-35 sand. Moment and fabric tensors were calculated to characterize the distribution. Contact normal distribution was initially oriented away from the loading direction, and upon compression the contact normal vectors oriented more in the loading direction to resist the load. Anisotropy of the fabric based on contact normal vectors increased dramatically until peak stress state was reached, then decreased to almost a constant value.

Quantitative particle characteristics described by this paper provides valuable insight into the fabric and failure mechanisms of granular material. Loading paths through granular material depend on particle shape, orientation, and contact, which are defined by the current study. Results from this analysis can be used in many future applications such as tracking particle kinematics at progressive loading, verifying numerical models, or input for force chain models, to name a few. This research provides a first step for future development of accurate micromechanical models that can eventually be used to design many different types of granular systems.

\subsection{Acknowledgements}

This material is partially funded by the US National Science Foundation (NSF) under Grant No. CMMI-1266230 and Office of Naval Research (ONR) grant No. N00014-11-1-0691. Any opinions, findings, and conclusions or recommendations expressed in this material are those of the authors and do not necessarily reflect the views of the NSF or ONR. This paper used resources of the Advanced Photon Source (APS), a U.S. Department of Energy (DOE) Office of 
Science User Facility operated for the DOE Office of Science by Argonne National Laboratory (ANL) under Contract No. DE-AC02-06CH11357. The SMT images presented in this paper were collected using the x-ray Operations and Research Beamline Station 13-BMD at Argonne Photon Source (APS), ANL. We thank Dr. Mark Rivers of APS for help in performing the SMT scans. We also acknowledge the support of GeoSoilEnviroCARS (Sector 13), which is supported by the National Science Foundation, Earth Sciences (EAR-1128799), and the DOE, Geosciences (DE-FG02-94ER14466).

The authors also thank David Fox from ARL, the DoD Productivity Enhancement, Technology Transfer and Training (PETTT) program as well as Chris Kung and Peter Raeth from Engility Corporation who, through the PETTT program, provided training and assistance in porting the code and making it more efficient.

\subsection{References}

Al-Raoush, R., 2007. Microstructure characterization of granular materials. Physica A: Statistical Mechanics and its Applications 377, 545-558.

Alshibli, K., Druckrey, A., Al-Raoush, R., Weiskittel, T., Lavrik, N., 2014. Quantifying Morphology of Sands Using 3D Imaging. Journal of Materials in Civil Engineering, 04014275.

Andò, E., Hall, S., Viggiani, G., Desrues, J., Bésuelle, P., 2012. Grain-scale experimental investigation of localised deformation in sand: a discrete particle tracking approach. Acta Geotechnica 7, 1-13.

Baruchel, J., Buffiere, J.-Y., Cloetens, P., Di Michiel, M., Ferrie, E., Ludwig, W., Maire, E., Salvo, L., 2006. Advances in synchrotron radiation microtomography. Scripta Materialia $55,41-46$.

Bloom, M., Russell, M., Kustau, A., Mandayam, S., Sukumaran, B., 2009. An X-ray computed tomography technique for the measurement of packing density in granular particles, Instrumentation and Measurement Technology Conference, 2009. I2MTC '09. IEEE, pp. 74-79.

Brunke, O., Brockdorf, K., Drews, S., Müller, B., Donath, T., Herzen, J., Beckmann, F., 2008. Comparison between $\mathrm{x}$-ray tube-based and synchrotron radiation-based $\mu \mathrm{CT}$, pp. 70780U-70780U-70712.

Cnudde, V., Boone, M.N., 2013. High-resolution X-ray computed tomography in geosciences: A review of the current technology and applications. Earth-Science Reviews 123, 1-17. 
Cox, M.R., Budhu, M., 2008. A practical approach to grain shape quantification. Engineering Geology 96, 1-16.

Druckrey, A., Alshibli, K., 2014. 3D Behavior of Sand Particles Using X-Ray Synchrotron Micro-Tomography, Geo-Congress 2014 Technical Papers, pp. 2814-2821.

Fonseca, J., O'Sullivan, C., Coop, M.R., Lee, P.D., 2013. Quantifying the evolution of soil fabric during shearing using directional parameters, Geotechnique, pp. 487-499.

Hall, S.A., Bornert, M., Desrues, J., Pannier, Y., Lenoir, N., Viggiani, G., BéSuelle, P., 2010. Discrete and continuum analysis of localised deformation in sand using X-ray \&\#956;CT and volumetric digital image correlation, Geotechnique, pp. 315-322.

Hasan, A., Alshibli, K.A., 2010. Experimental assessment of 3D particle-to-particle interaction within sheared sand using synchrotron microtomography, Geotechnique, pp. 369-379.

Kanatani, K.-I., 1984. Distribution of Directional Data and Fabric Tensors. Int. J. Engng Sci. 22, 149-164.

Oda, M., 1972. INITIAL FABRICS AND THEIR RELATIONS TO MECHANICAL PROPERTIES OF GRANULAR MATERIAL. Soils and Foundations 12, 17-36.

Oda, M., Nemat-Nasser, S., Konishi, J., 1985. STRESS-INDUCED ANISOTROPY IN GRANULAR MASSES. Soils and Foundations 25, 85-97.

Rivers, M.L., Citron, D.T., Wang, Y., 2010. Recent developments in computed tomography at GSECARS. SPIE 3, 780409-780409.

Theocharis, A., Vairaktaris, E., Fu, P., Dafalias, Y.F., 2014. Comparison of fabric tensors for granular materials, Geomechanics from Micro to Macro. CRC Press, pp. 153-158.

Thompson, K.E., Willson, C.S., Zhang, W., 2006. Quantitative computer reconstruction of particulate materials from microtomography images. Powder Technology 163, 169-182.

Tordesillas, A., Pucilowski, S., Tobin, S., Kuhn, M., R., Andò, E., Viggiani, G., Druckrey, A., Khalid, A., 2015. Shear bands as bottlenecks in force transmission. EPL (Europhysics Letters) 110, 58005.

Viggiani, G., Andò, E., Jaquet, C., Talbot, H., 2013. Identifying and following particle-toparticle contacts in real granular media: An experimental challenge. AIP Conference Proceedings 1542, 60-65.

Vlahinić, I., Andò, E., Viggiani, G., Andrade, J., 2014. Towards a more accurate characterization of granular media: extracting quantitative descriptors from tomographic images. Granular Matter 16, 9-21.

Yang, D.S., Li, X., Yu, H.S., 2014. Fabric evolution of 3D granular materials in rotational shear, Geomechanics from Micro to Macro. CRC Press, pp. 85-90.

Yimsiri, S., Soga, K., 2010. DEM analysis of soil fabric effects on behaviour of sand, Geotechnique, pp. 483-495. 


\section{Figure Captions}

Fig. 1. Steps of particle identification process

Fig. 2. Example of three types of voxel connectivity

Fig. 3. Voxel surface of a particle to compute surface area (axis units are in pixels)

Fig. 4. Example 2D PCA analysis on 6810 pixels of a particle cross section (axis units are pixels)

Fig. 5. Illustrative example in $3 \mathrm{D}$ identifying particle global orientation and principal length.

Fig. 6. Particle size distribution of 3400 particles of ASTM 20-30 sand based on SMT image analysis.

Fig. 7. Illustration of contact voxels between two particles (axis units are in pixels).

Fig. 8. Normal and tangent contact vectors between two particles (axis units are in pixels).

Fig. 9. Flow chart of the code

Fig.10. Axial strain versus principal stress ratio of axisymmetric triaxial compression experiment on F-35 sand.

Fig. 11. Distribution of fabric descriptors for axisymmetric triaxial compression experiment on F-35 sand at $0,2,5$, and $17.5 \%$ axial strains.

Fig. 12. Evolution of $F_{I I}$ during axisymmetric triaxial test of F-35 sand. 


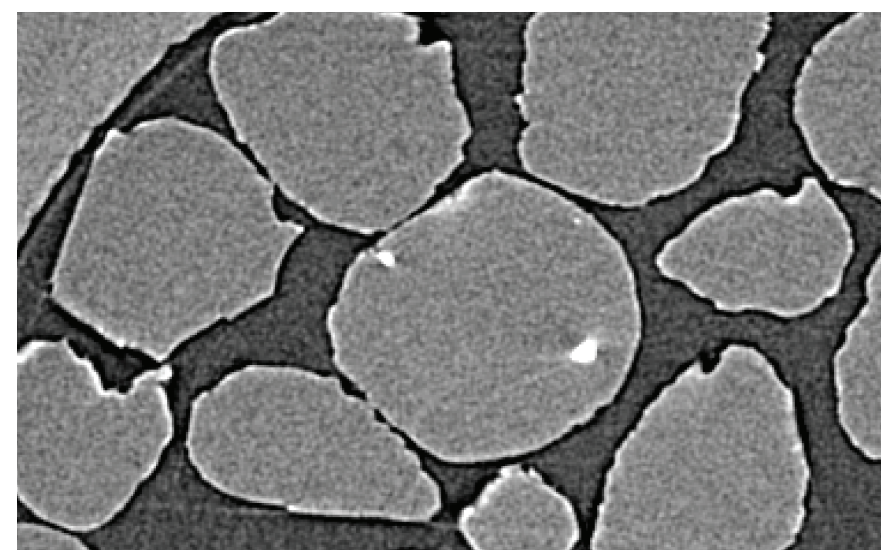

(a) Original SMT greyscale image

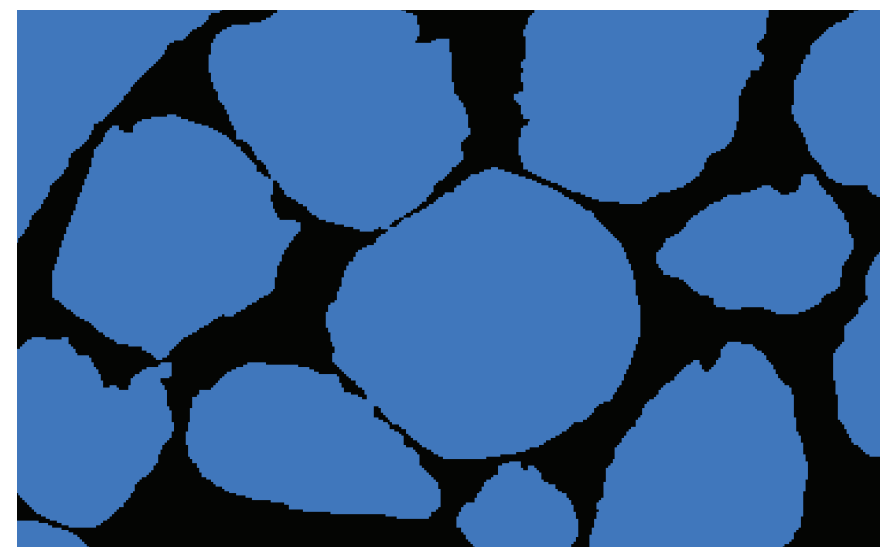

(c) Binary image with intact contact point:

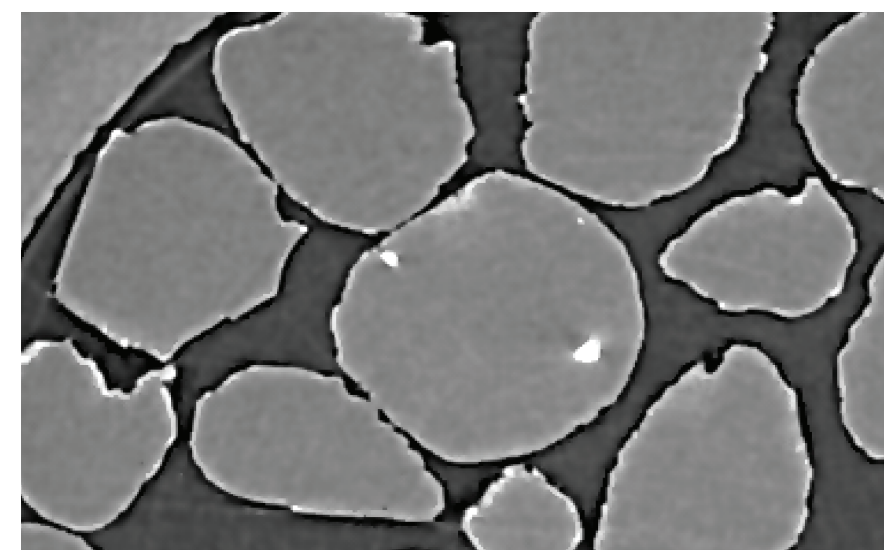

(b) Filtered SMT greyscale image

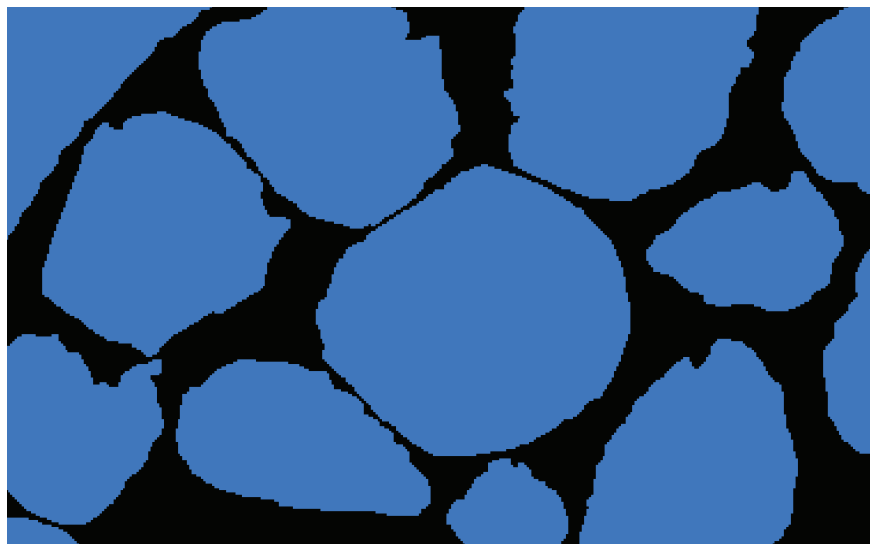

(d) Binary image with separated particles

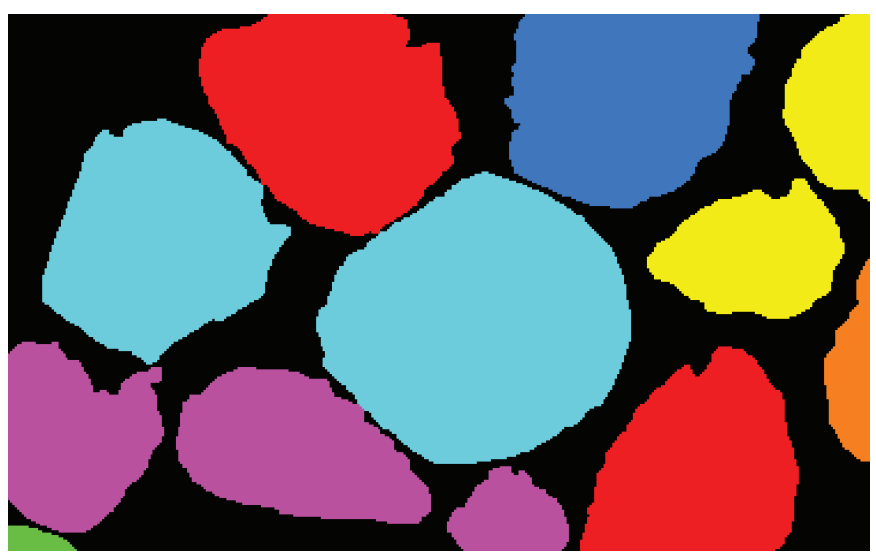

(e) Labeled particles with intact contact points 


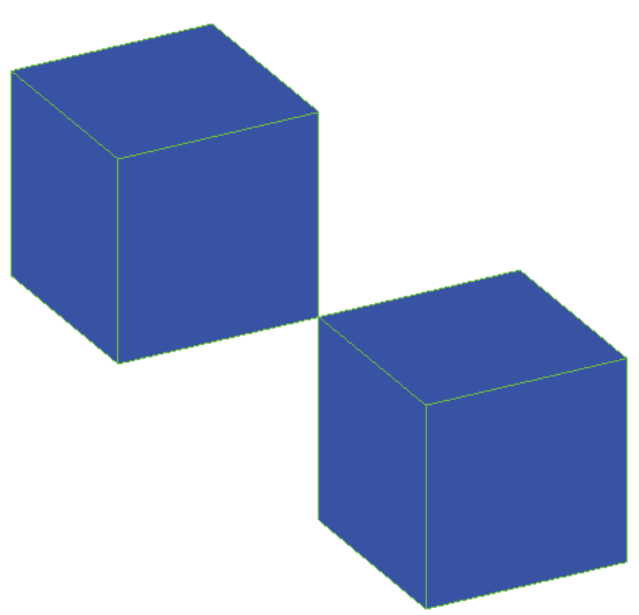

(a) Face-connected voxels

(b) Edge-connected voxels

Figure 2

(') (
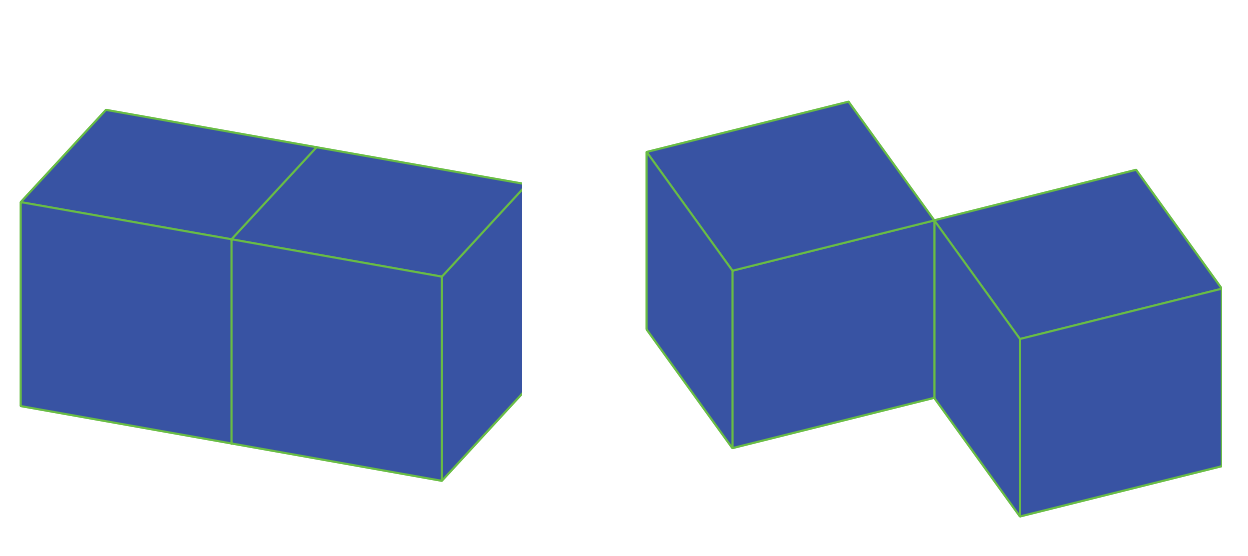

ls

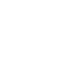

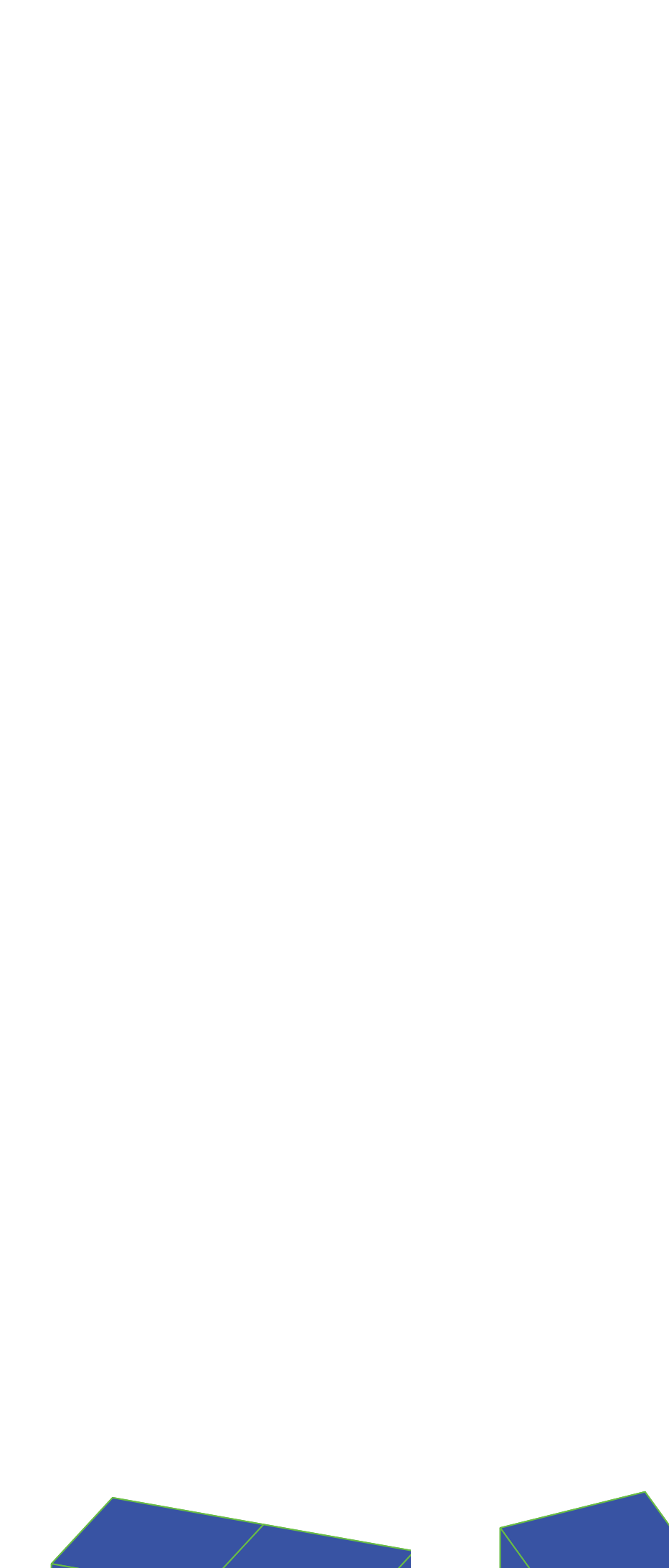

- 0




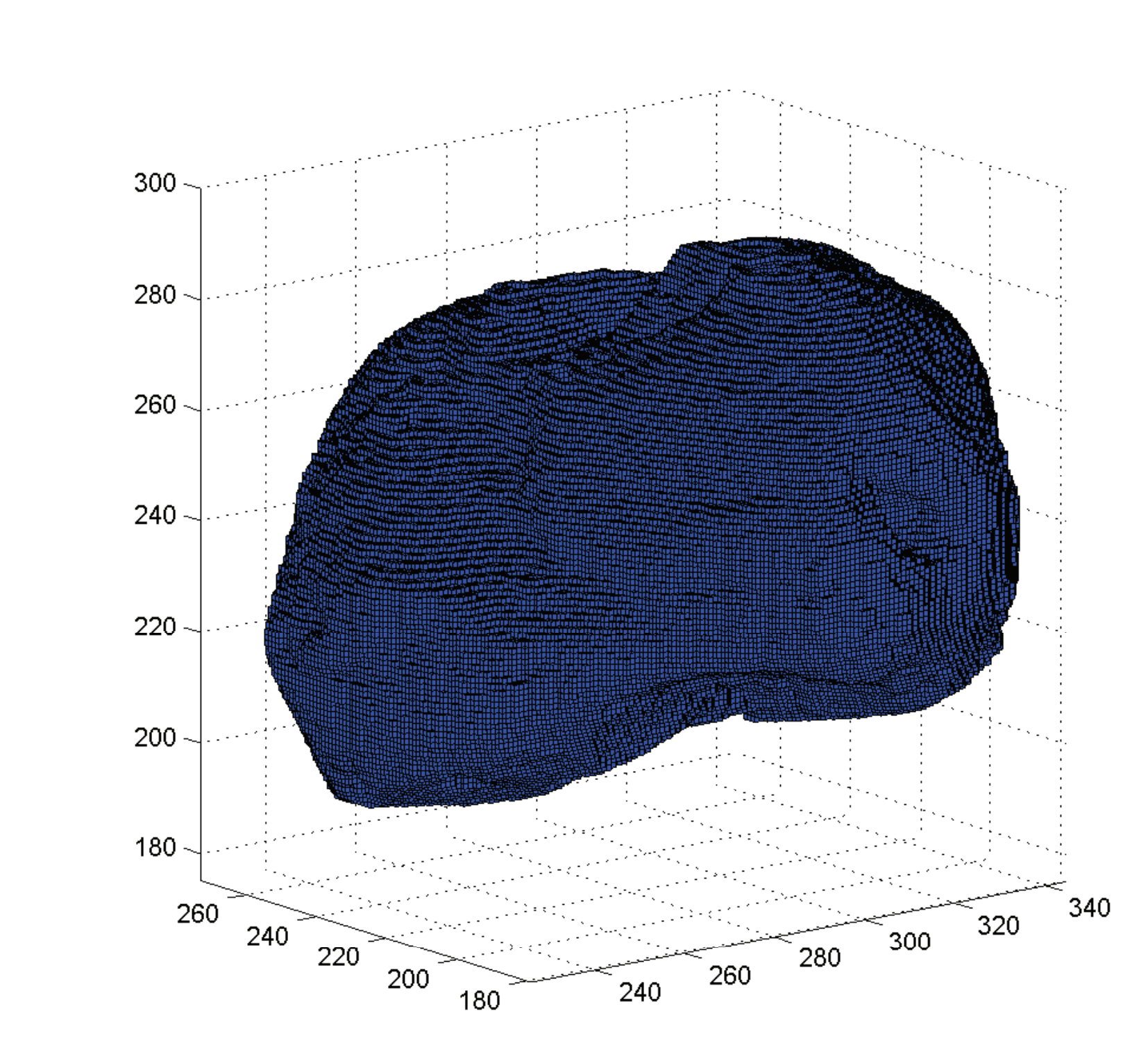

Figure 3

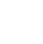

. 


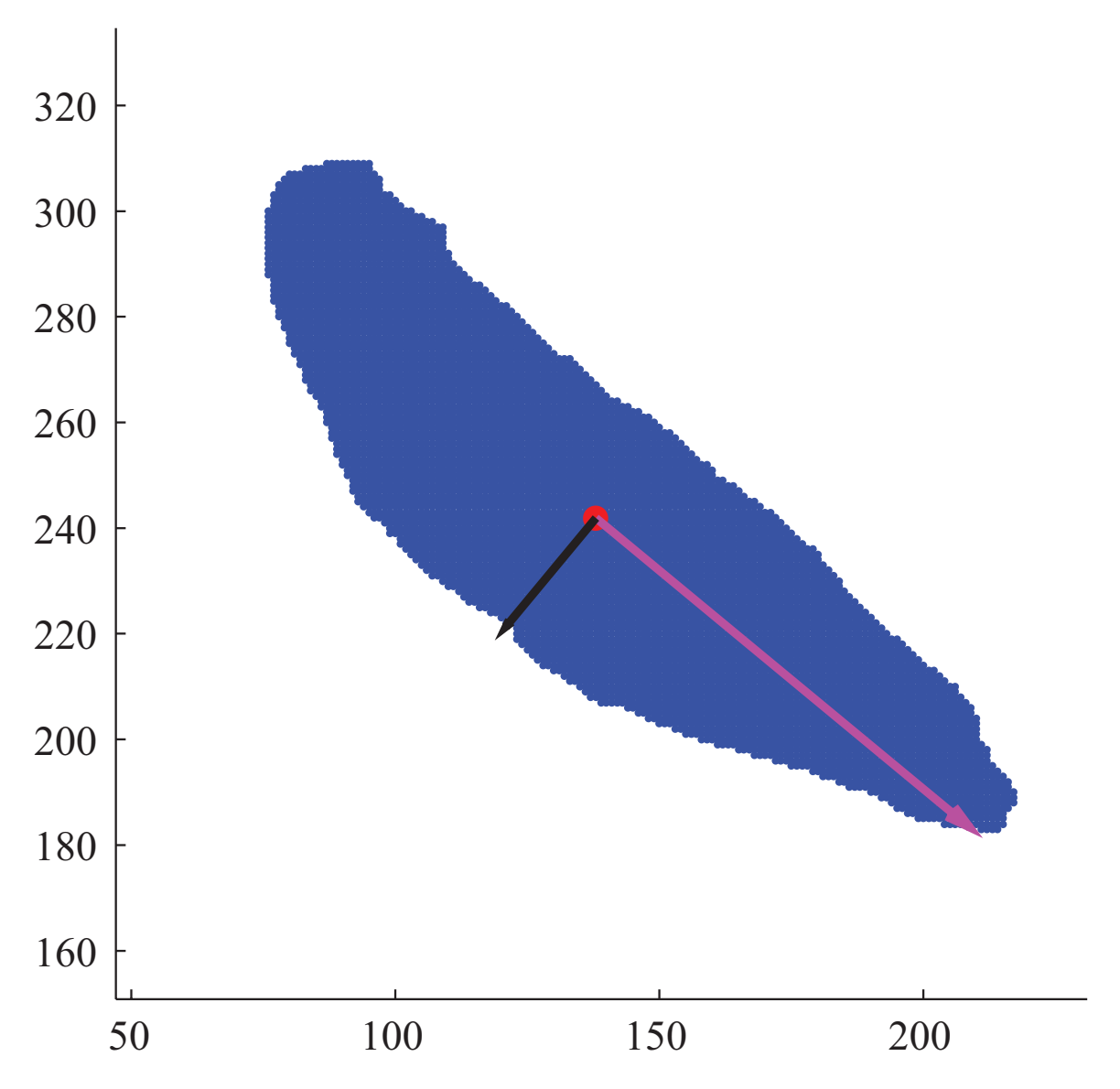

Figure 4

2) 


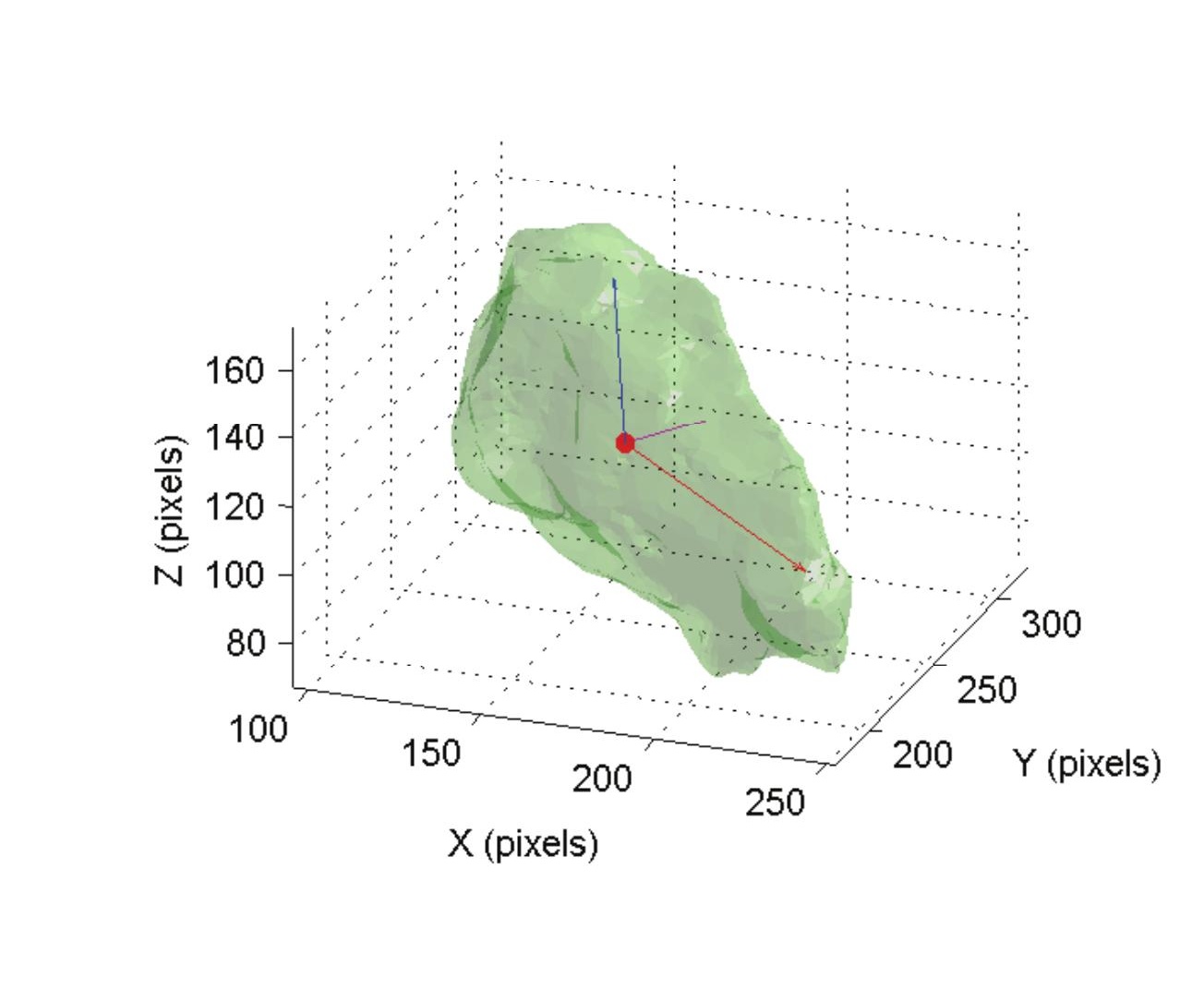

Figure 5

\section{5}

(1)

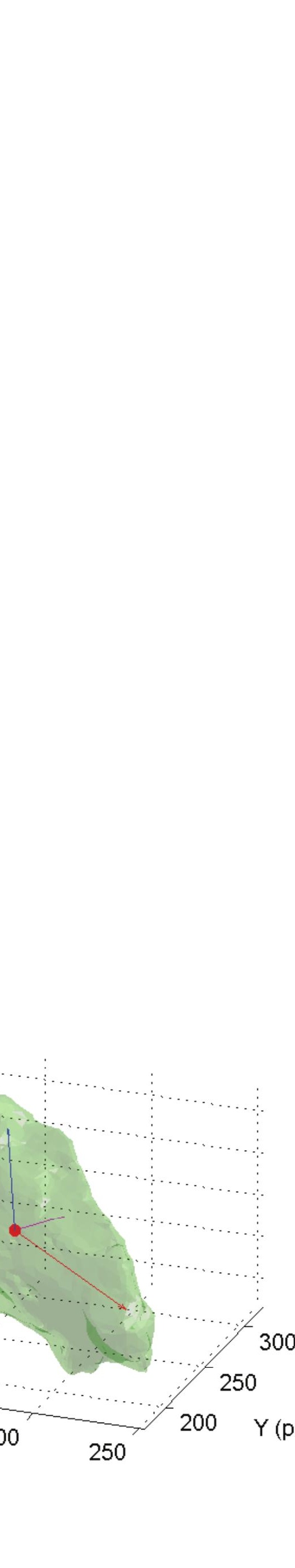




\title{
Figure 7
}

(a) Two particles in contact

(a) Two particles in con

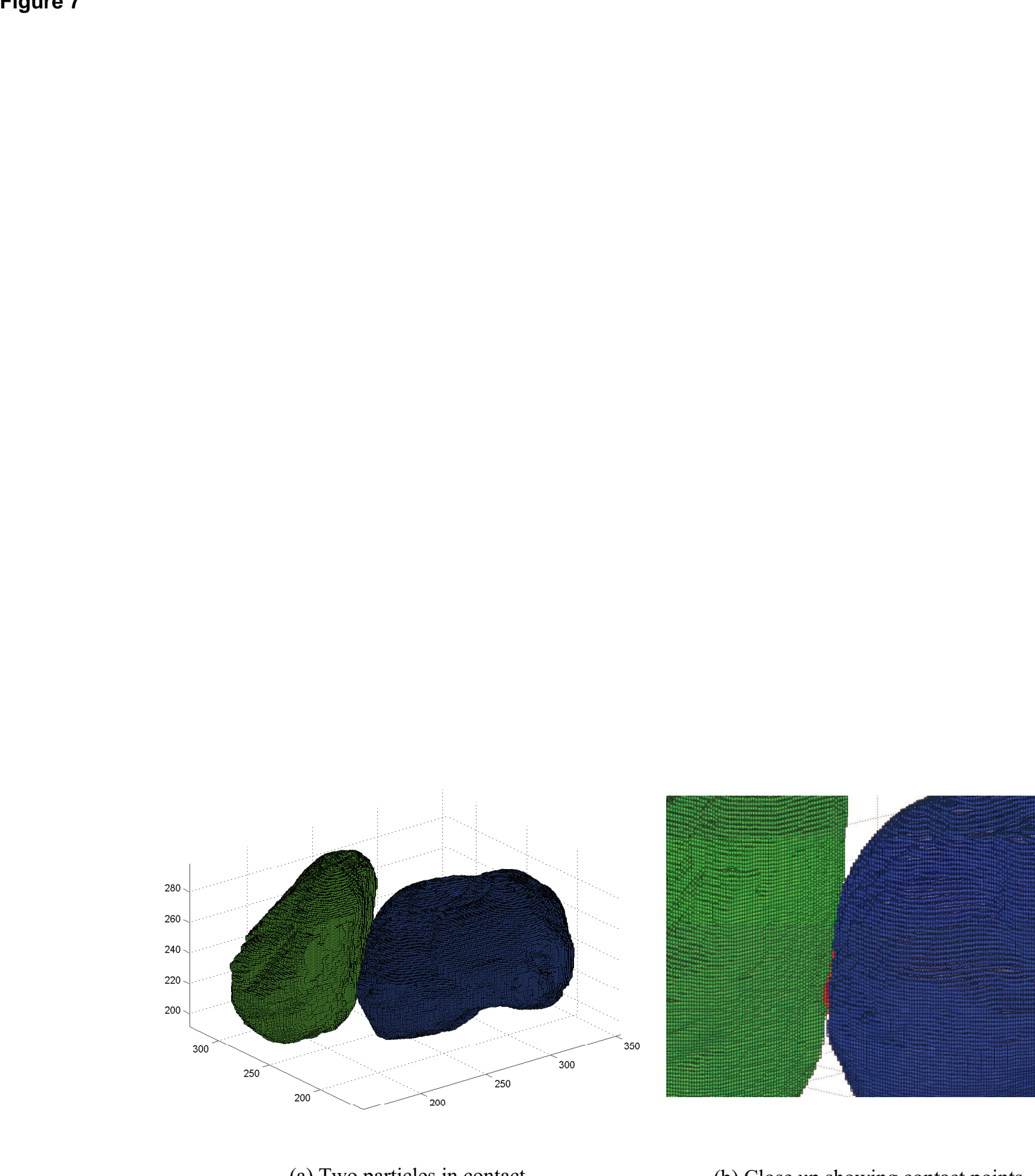

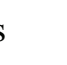

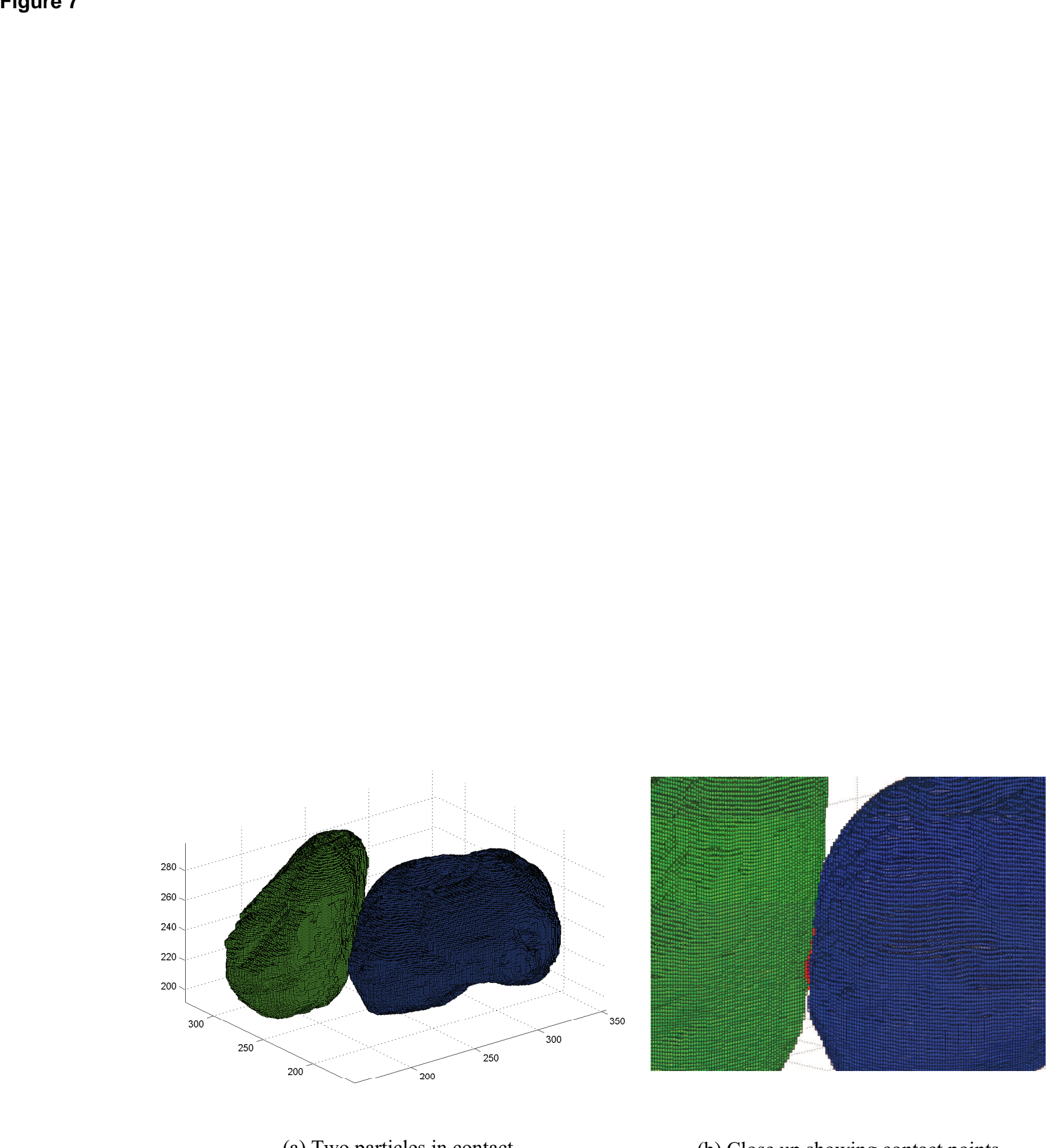

(b) Close up showing contact points

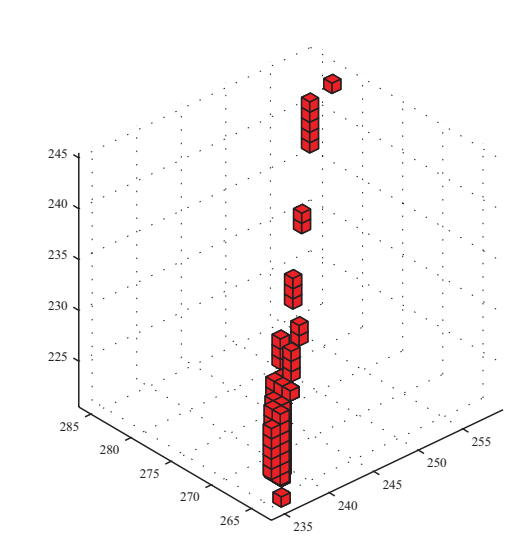

(c) Contact points between the two particles

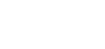

$=0$ 
Figure 8

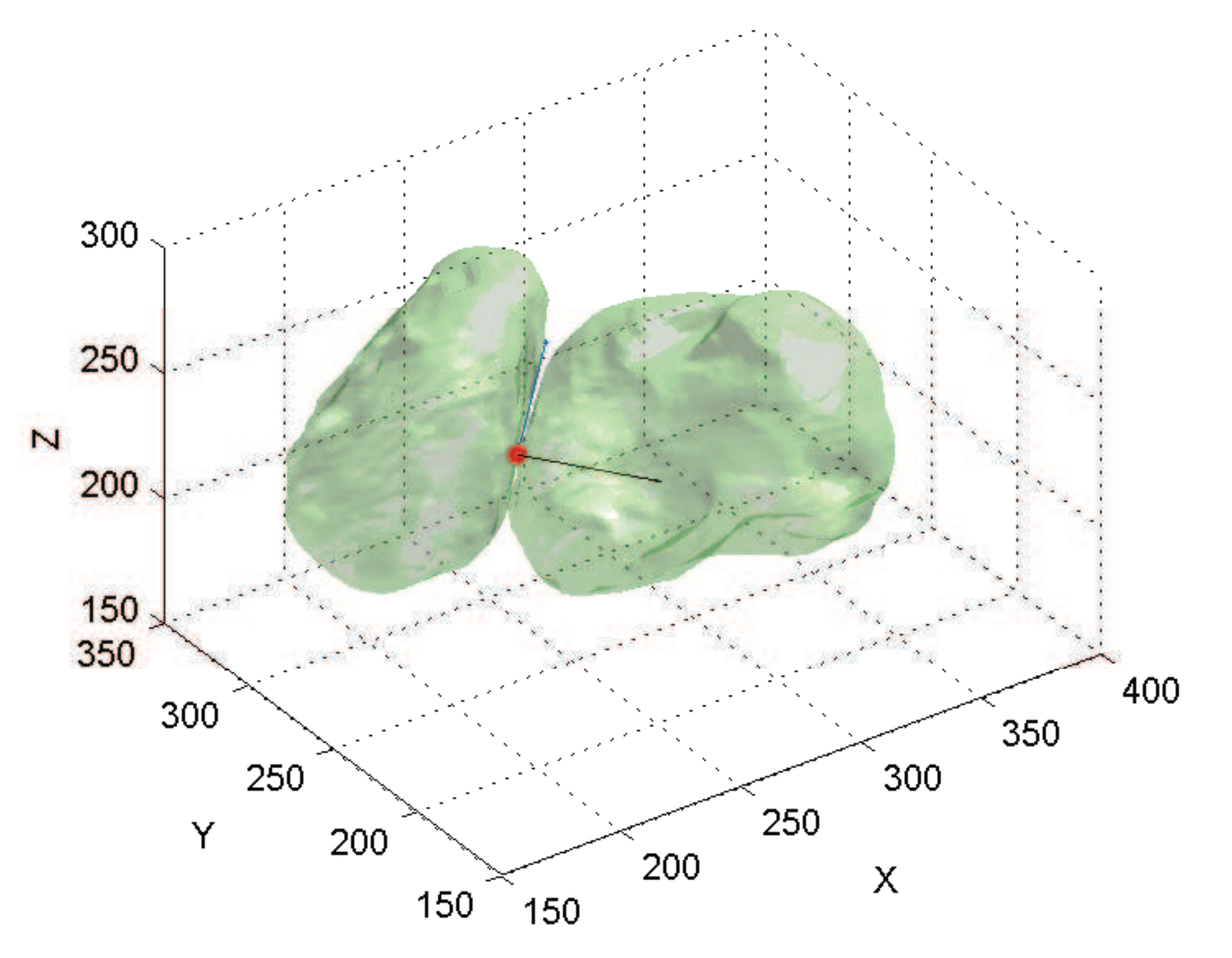

300

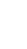

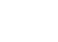


Figure 10

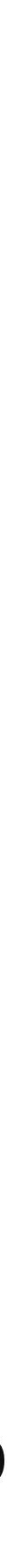

Figure 10
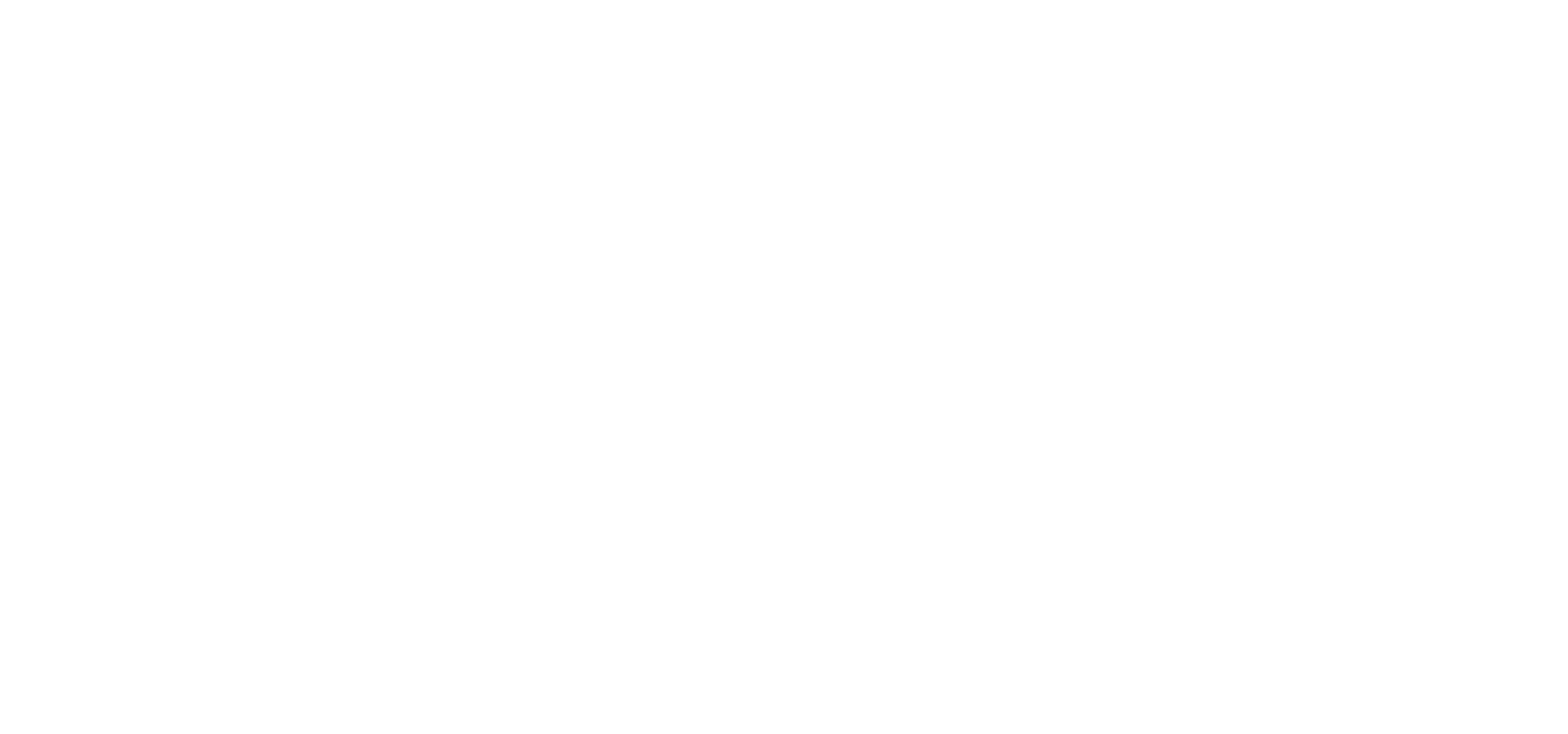
Figure 12

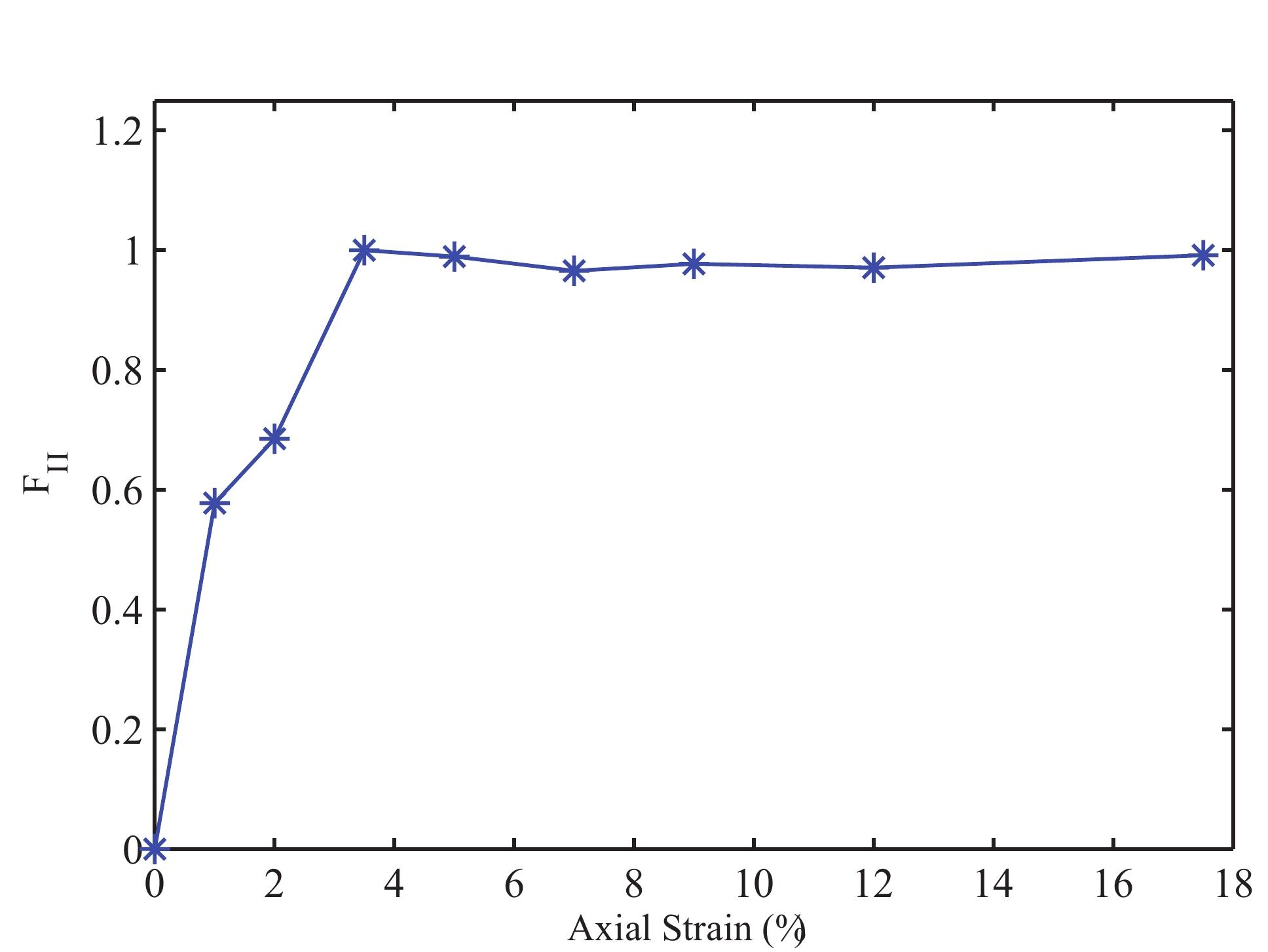

Axial Strain (ox)

Figure 12 -

.

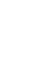

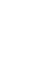
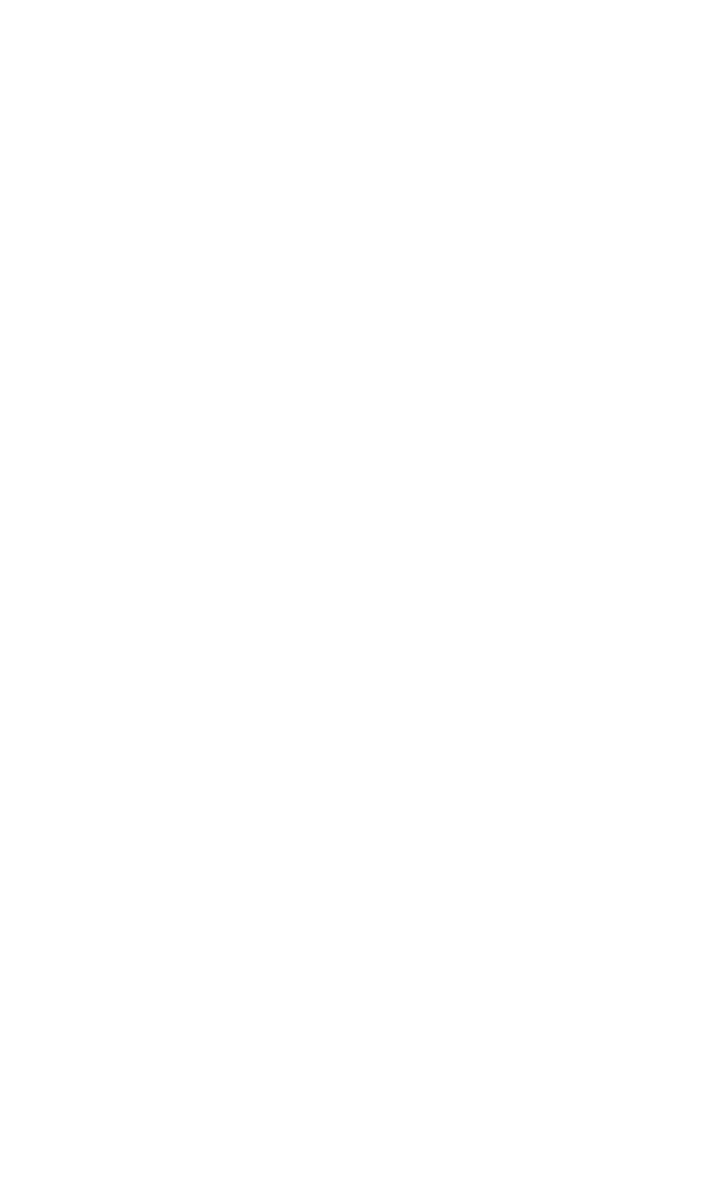